\title{
Effective use of simple computer programmes and clay models to demonstrate the planning and operative steps for teaching and presentations
}

\author{
Rahul Krishnarao Patil, Gopal Malhotra, Venugopal Srinivasan, Ahmed Osama Abdul Hamid, \\ Mahil Cherian, Ashok Raj Koul ${ }^{1}$ \\ Department of Plastic and Reconstructive Hand and Micro-Vascular Surgery, Khoula Hospital, Muscat, Oman, ${ }^{1}$ Department of \\ Plastic and Reconstructive Hand and Micro-Vascular Surgery, Sparsh Hospital, Bengaluru, Karnataka, India
}

Address for correspondence: Dr. Rahul Krishnarao Patil, Department of Plastic and Reconstructive Hand and Micro-Vascular Surgery, Khoula Hospital, Al Wuttayya, PC 116, Muscat, Oman. E-mail: doctrahul@yahoo.co.in

\begin{abstract}
Background: Presenting and demonstrating a surgical procedure in the current era is difficult without good intraoperative pictures and videos. A long, complex, multi-staged surgery is better illustrated by detailed intraoperative images at various stages. Although desirable, it may be difficult due to various reasons. Material and Methods: A simple method of preparing illustrations with pictures/diagrams created on PowerPoint and clay models to recreate the missing links in clinical photographs has been proposed. Results: It is a simple technique with a moderate learning curve. Once familiar with technique, one can effectively use the technique to convey the details in much more clear manner. Conclusion: It is a simple and effective way of communicating through digital images, and gives the audience a 3 dimensional idea about the concept.
\end{abstract}

\section{KEY WORDS}

Clay models in plasticine surgery; photography; presentations in plastic surgery; teaching and patient education; use of clay models for presentations

\section{INTRODUCTION}

utting up a presentation just ahead of a conference is often an intimidating task for a busy surgeon. High-quality clinical pictures and clips have become an integral part of today's scientific presentations. Unless the photographs and videos are obtained in a standard manner and the data are stored carefully,

\begin{tabular}{|l|l|}
\hline \multicolumn{2}{|c|}{ Access this article online } \\
\hline Quick Response Code: & Website: \\
\hline & www.ijps.org \\
\hline & DoI: \\
\hline
\end{tabular}

retrieving good quality images at short notice becomes difficult. Three-dimensional image reconstruction as computer-assisted designing in craniofacial and orthognathic surgical planning ${ }^{[1,2]}$ and software programmes to create pre- and post-operative images for aesthetic procedures are currently in use. Generally, these techniques use highly, sophisticated software programmes

This is an open access article distributed under the terms of the Creative Commons Attribution-NonCommercial-ShareAlike 3.0 License, which allows others to remix, tweak, and build upon the work non-commercially, as long as the author is credited and the new creations are licensed under the identical terms.

For reprints contact: reprints@medknow.com

How to cite this article: Patil RK, Malhotra G, Srinivasan V, Abdul Hamid AO, Cherian M, Koul AR. Effective use of simple computer programmes and clay models to demonstrate the planning and operative steps for teaching and presentations. Indian J Plast Surg 2016;49:185-90. 
that are expensive and need trained professionals for handling them. No such special programmes are commonly used for routine reconstructive procedures.

We present an easy way of creating sketches and models that can be used for the PowerPoint presentation, teaching sessions and other academic purposes from easily available materials and software programmes. This technique is simple, rapid, user-friendly and uses freely available software programmes. They are very effective in conveying the idea/concept to the audience.

\section{MATERIALS AND METHODS}

The Microsoft Office ${ }^{\circledR}$ software available in Windows $7^{\circledR}$ system was used for drawing the sketches. The other set of the models was prepared from the plasticine modelling clay, which is used in schools by the children to prepare clay models and is widely available in the market.

Microsoft Office is available in most of the computers. The basic features of the programme have not undergone major changes since 1997. 'Microsoft PowerPoint' programme was used for this purpose. Shapes are drawn with 'Freehand line drawing' tool [Figure 1a and b]. One can use scrollbar or mouse; later is more comfortable for handling. After creating the shape and the colours, borders and three-dimensional effects can be added as necessary with picture editing options [Figure 1c-e]. Arranging shapes in an overlapping manner and using variable colours and transparency for each shape can create a layered appearance [Figure 1f-h]. The drawing can be saved as an image or can be copied and pasted in another slide (it remains editable) intermittently so that further changes can be made without losing the original progress. An illusion of animation to some extent is possible with windows movie maker programme (freely available with original windows) [Figure $1 \mathrm{i}-\mathrm{k}$ ] where sequential images can be converted into a movie that can be saved in various formats [Figure 11]. These videos can then be easily added to a PowerPoint. The learning curve is mild to moderate.

Plasticine clay is another way of creating illustrations of three-dimensional shapes. ${ }^{[3,4]}$ It is normally used by students for preparing different models. One can easily use it to create models, giving it a natural three-dimensional structures and feel. This clay is available in different colours, can be moulded easily with hands at room temperature, retains its shape, does not mix with each other and hence most of it can be reused. Different colours and layers can be used for demonstrating various structures/tissues. The colour combination theme is kept constant throughout the series for easier understanding. Normally, colours matching natural colours of structures are preferred, e.g. muscle can be red while skin graft can be brown. Once the models are created, standardised photographs in appropriate conditions will help one demonstrate various stages as digital images.

\section{Example 1}

Microsoft Word programme and clay models were used to demonstrate a pre-laminated radial forearm free flap for nasal reconstruction. For convenience of description, the computer-generated images and the clay model pictures are placed next to each other with the same figure numbers. This also helps in easy comparison between the two methods [Figures 2-6]. These images were used for a presentation to connect the missing links in clinical pictures.

\section{Example 2}

While writing an article, we had difficulty in demonstrating the possible abnormal patterns of pedicle orientation while doing a free tissue transfer due to lack of images. Situation was managed by similar schematic pictures [Figures 7 and 8]. Continuous irrigation of anastomosis site with heparin solution using a cannula in one of tributaries was similarly demonstrated [Figure 9] (illustrations re-used with permission from Medknow Publications). ${ }^{[5]}$

\section{DISCUSSION}

\section{A picture speaks thousand words ${ }^{[6]}$}

Clinical photographs and scientific presentations have undergone major changes in the recent past. ${ }^{[2]}$ With widespread availability of the user-friendly gadgets such as digital cameras and mobile phones, improved optics, ease of transferring, processing and storing images, use of high-quality images for presentations has become a standard practice..$^{[1,7,8]}$ Although on one side the instrumentation is improving, it is not immune to data loss. Accidental deletion, loss during transfers, software crashes to viruses, anything can damage meticulously stored data. In addition, ease of doing things brings casualness to it and often if not always, the importance of obtaining, segregating and storing images is ignored. 


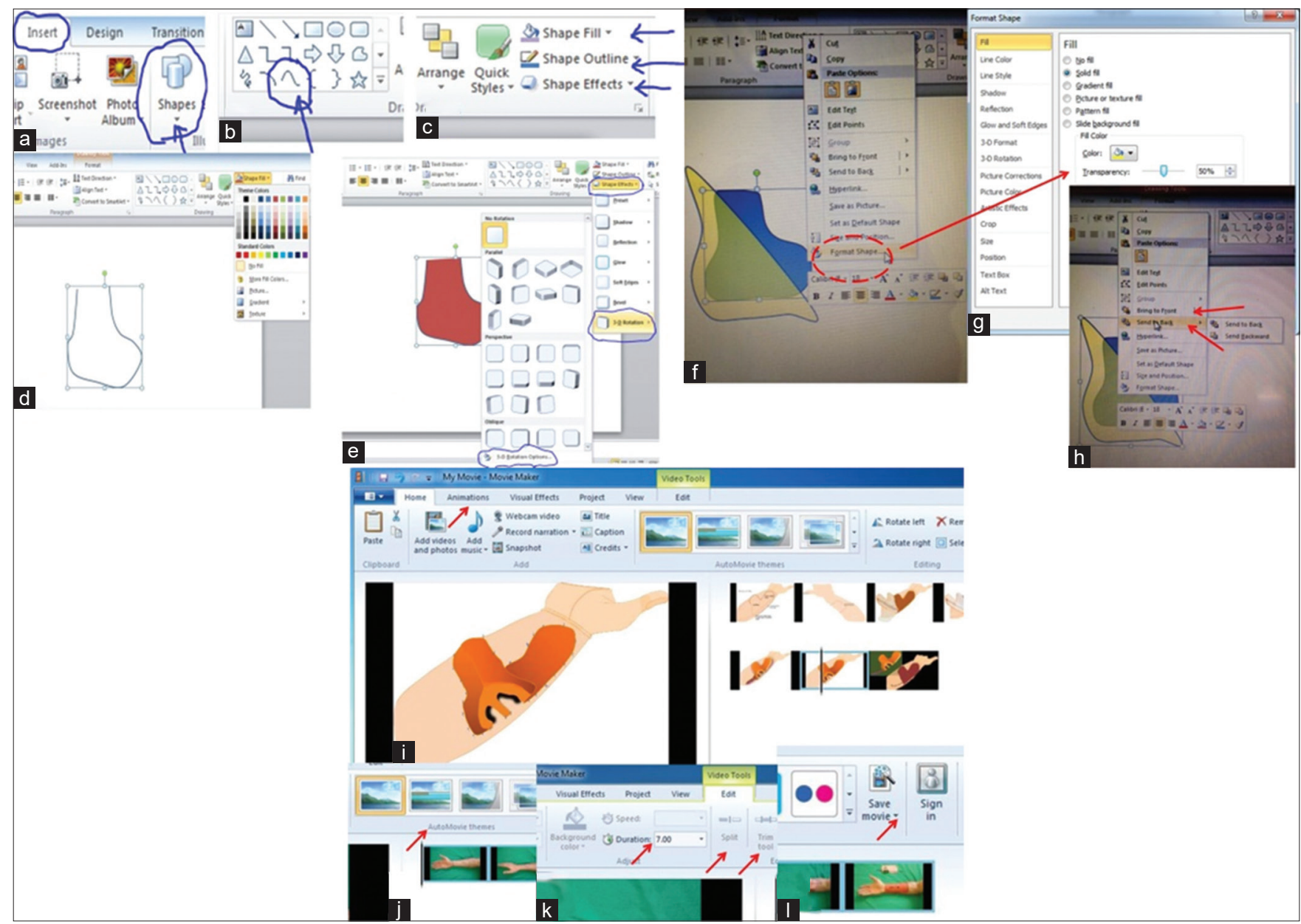

Figure 1: Guide regarding use of the PowerPoint tools for drawing pictures. (a) Selection of the option of inserting free shapes to the slide. (b) Selection of the freehand line drawing tool to draw the desired shape. (c) Once the shape is complete, one can choose/alter the colours of fill, margins, etc., (d) For further modification, one can explore the necessary window in more details. (e) The three-dimensional effects can be selected from the shape effects option on the toolbar. (f) After selecting the shapes, right clicking on the shapes will open a window with editing options; when one chooses the format shape option, it will take one to a new window. $(\mathrm{g})$ Where the transparency of each shape, the colour can be modified. (h) The pictures can be arranged in a proper sequence as necessary by selecting the 'bring to front' or 'send back' options in the window after selecting and right clicking over a particular shape so that shape with different transparencies will be seen as layers. (i) The 'movie maker' programme can be selected from the start up menu (if necessary by going to the all programmes option). After opening the movie maker, the pictures can be dragged into or selected from appropriate folders. It can be animated either by selecting one of the animations option, auto themes or by dragging the images to overlap them (j). (k) The exposure to each film can be selected and controlled to create specific length of movie, and (I) a movie created in such a way can be saved in appropriate format by selecting the save movie option

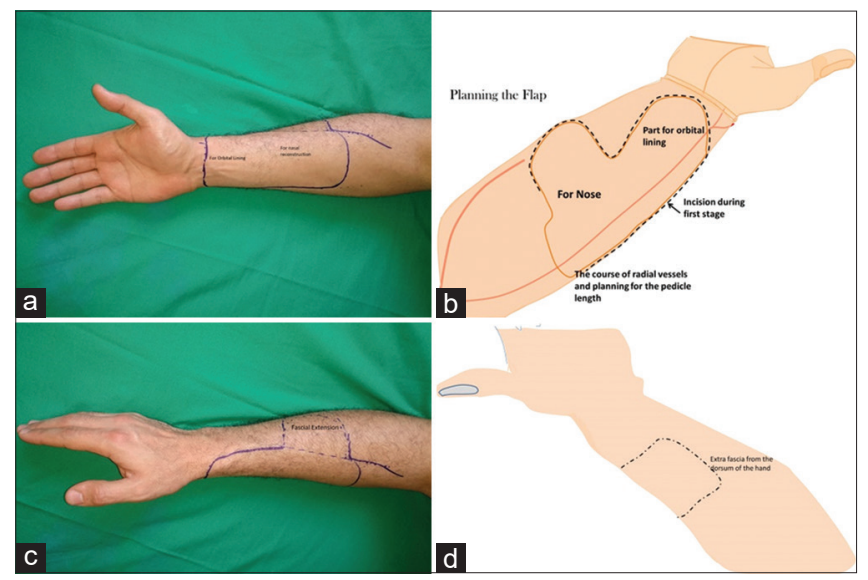

Figure 2: $(a$ and $b)$ Demonstrating the planning of the procedure by marking the flap on volar surface of the forearm. An extra part of the flap was planned for lining the orbital cavity. (c and d) The facial extension of the flap was planned over the dorsal aspect of the hand going around the radial border of the forearm

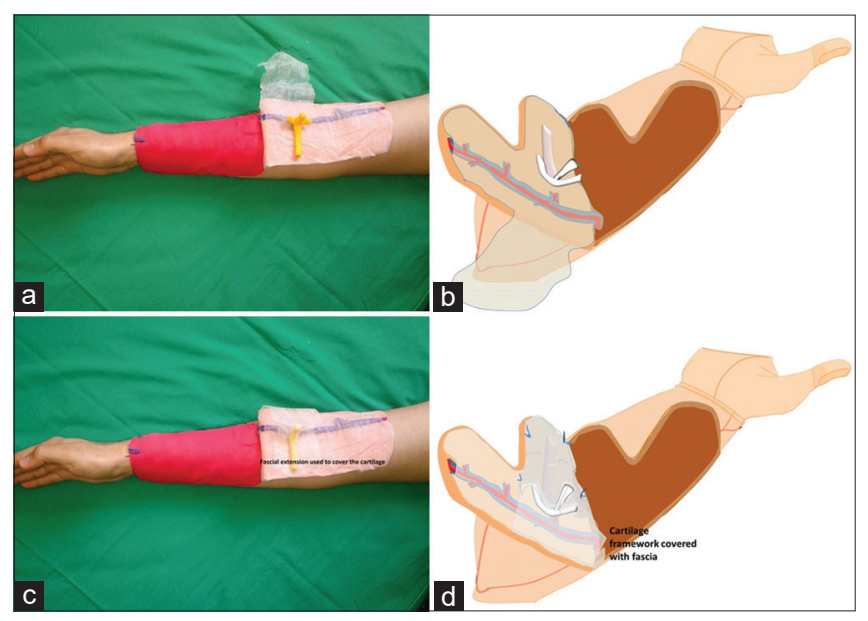

Figure 3: $(a$ and $b)$ The flap with necessary fascia was raised and cartilage has been shaped and placed over the fascia. (c and d) The fascia was used to cover the cartilage as an additional vascular layer

Indian Journal of Plastic Surgery May-August 2016 Vol 49 Issue 2 


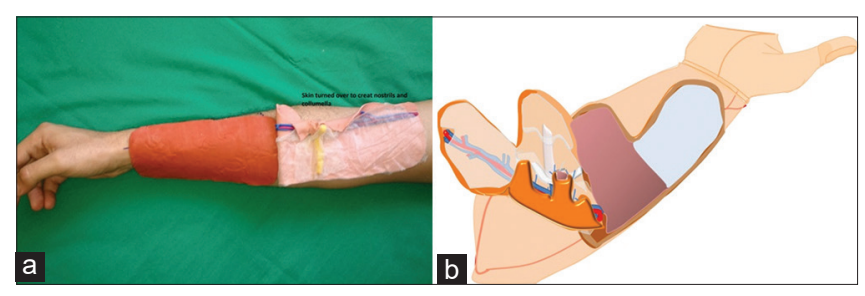

Figure 4: $(a$ and $b)$ The skin part of the flap was used to line the collumella and the nostril openings
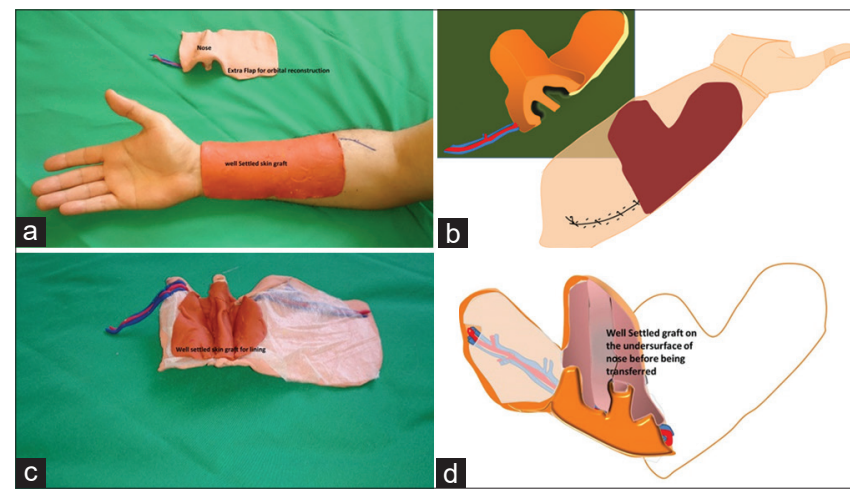

Figure 6: $(a$ and b) 4 weeks following the in initial surgery when the flap and the graft had settled well the flap was raised and transferred to the recipient site. (c and d) The undersurface of the flap showing the well-accepted skin graft for future nasal lining

Personal preferences contribute as well; stopping intermittently for stepwise clinical pictures and obtaining them in standard format may not appeal to some surgeons. Sometimes, situations would not permit the same (Example 2 above) ${ }^{[9,10]}$ Even now, many senior surgeons are not comfortable with the ever-changing technology. Availability of an extra person to willingly take photographs in OT can also be an issue. Except in teaching institutions, busy surgeons commonly face these problems. Hence, during an extended procedure, either no pictures are taken or multiple persons would have taken images in their cell phones. The picture orientation, lighting and the overall quality of the image are often ignored in such instances. ${ }^{[8,11]}$

When images are unavailable/lost, the proposed method provides a unique way of re-creating the steps and giving at least a schematic idea to the audience for easier understanding. The three-dimensional qualities of the clay models are more appealing and also to an extent these can be incorporated into computer drawings. These models/images can be useful in many ways.

1. These models and images can serve to link the missing steps in a multi-staged reconstructive procedure when some of the images are missing

2. It is said that one of the key attributes of a plastic surgeon is to be able to imagine and conceive

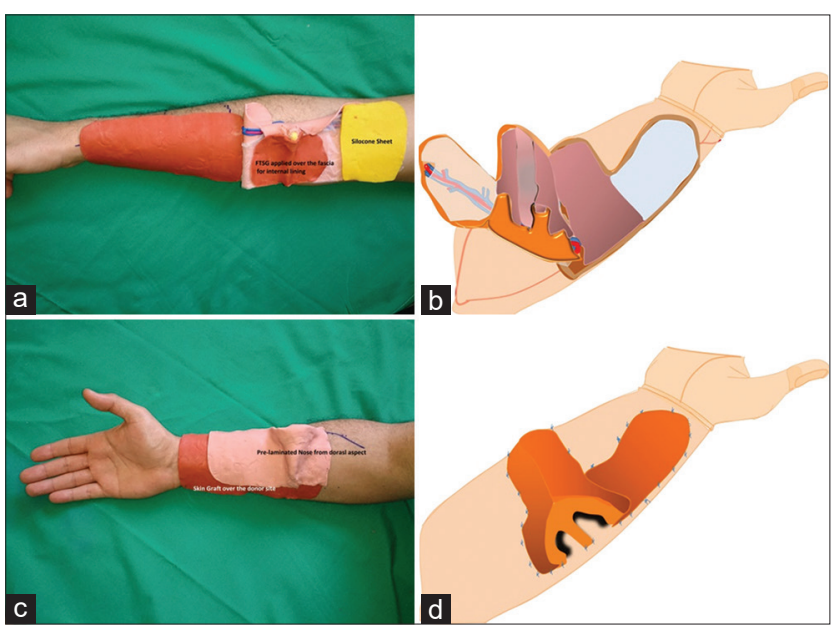

Figure 5: ( $a$ and b) Full thickness graft was used to cover the fascia that will form the inner lining of nose in future. Donor site was covered with skin graft and silicon sheet was placed over the extra part of the flap to prevent it from getting adherent to the bed. (c and d) The nose thus pre-laminated was packed and placed back over the donor site

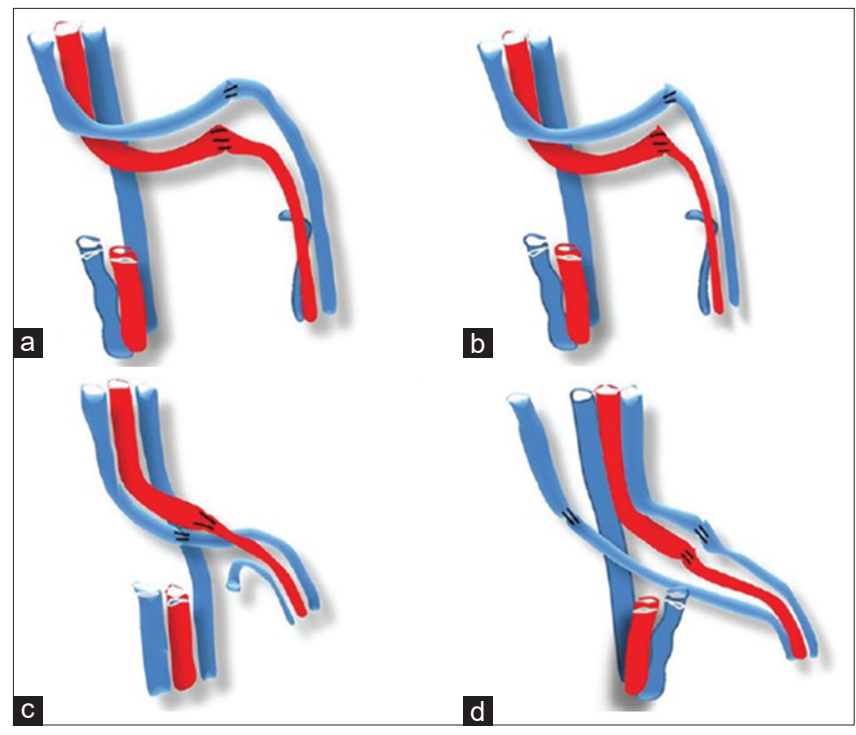

Figure 7: (a-d) Various pattern of lie of the pedicle, the lie shown in 'd' is preferable while other may have potential chance of kink and blockage by pressure due to overlapping

the result in mind before actually operating on a patient. The speciality if so vast that the challanges may vary from a relatively superficial surface defects to a composite defect needing lining, cover, supporting structures and sometimes even motor function. As demanded by time, speciality has seen evolution from multi-staged reconstruction of composite defects to single-stage reconstruction with pre-laminated and prefabricated flaps, transferred by microsurgery. These models can be used for teaching the complex designing of these flaps and even to a great extent help in the pre-surgical planning. Complex procedures where 


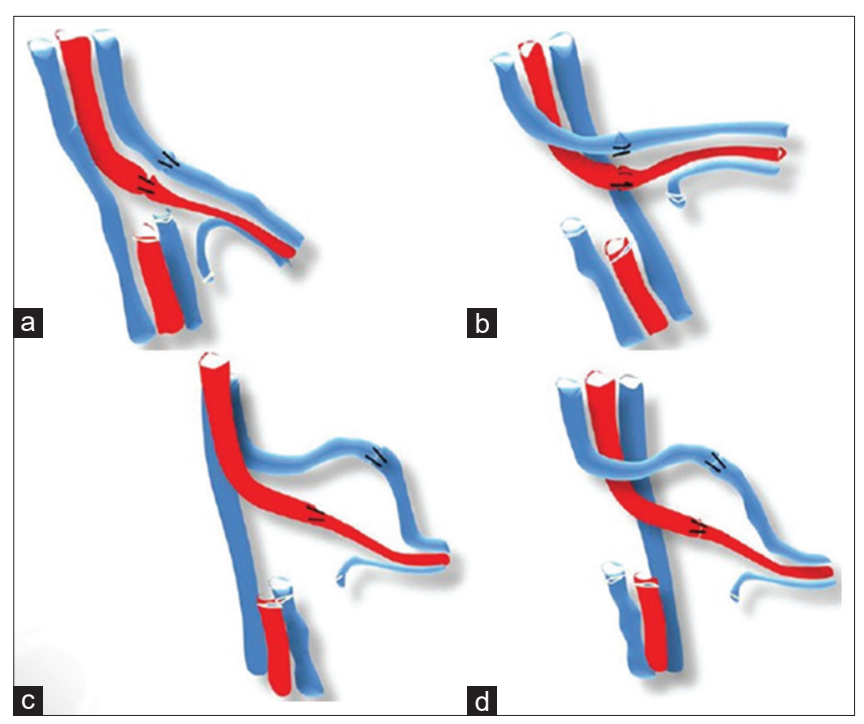

Figure 8: (a-d) Another set of possible pedicle orientations, 'a' is acceptable position while others can be potentially dangerous

three-dimensional orientations are necessary such as penile reconstruction, head and neck reconstruction and even the ear reconstruction can be explained and understood better with these models

3. These models can also be helpful in educating the patient about the complexity of the procedure this is very important especially in current litigation prone society for informed consent. It is especially necessary for making them understand the importance of each step in intra- and post-operative period and how a small complication can either downgrade the expected result or; prolong the staged procedure. Images are also handy when clinical pictures cannot be shown due to privacy or fear-related issues

4. Complex reconstructions, especially in head and neck and limbs have surgeons from different specialities actively participating in the procedure sequentially. Unless routinely involved in procedures and planning, surgeons belonging to other specialities may not be aware of the intricate points such as location and lie of the pedicle, perforators to the skin paddle, need for careful handeling, delicate vessel anatomy and protecting them during fixations. A model would help the colleagues understand and anticipate things rather than having difficulties during the procedure, thus saving valuable (sometimes ischemia) time.

We have used the simplest and widely available software programme. Drawing pad and pen can be used for with more control for effectively drawing shapes, but it involves an additional cost. Advanced programmes such

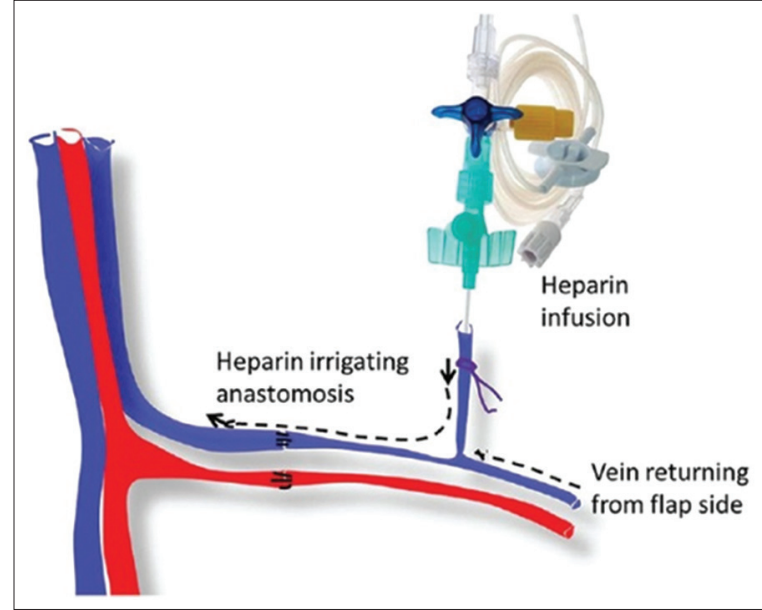

Figure 9: A special situation when the vein is not healthy, continuous heparin irrigation of the anastomosis can help in avoiding possible complications

as 'Adobe Photoshop' and 'CorelDraw' also can be helpful in similar ways, but one would need more proficiency in handling them. Proper animation of pictures and drawings also needs higher software support and professional help and has not been available with us currently. We now routinely use this technique during demonstrations of specific steps, anatomy of flaps as and when necessary. ${ }^{[5,12]}$

Intraoperative photographs are the gold standard and the proposed method is not an alternative. It is just a way of creating missing links for lost/misplaced pictures for a PowerPoint presentation in an emergency situation. Other applications of the technique as discussed above are worthwhile.

\section{CONCLUSION}

This easy and innovative way of demonstrating the surgical steps is helpful for interactive learning during classes, lectures, presentations, pre-surgical planning as well as patient education.

\section{Financial support and sponsorship}

Nil.

\section{Conflicts of interest}

There are no conflicts of interest.

\section{REFERENCES}

1. Lo LJ, Chen YR. Three-dimensional computed tomography imaging in craniofacial surgery: Morphological study and clinical applications. Chang Gung Med J 2003;26:1-10.

2. Dai J, Wang X, Hu G, Shen SG. A new method to move mandible Indian Journal of Plastic Surgery May-August 2016 Vol 49 Issue 2 
to intercuspal position in virtual three-dimensional orthognathic surgery by integrating primary occlusion model. J Oral Maxillofac Surg 2012;70:e484-9.

3. Pottery and clay modeling, published by research and education association. Available from: https://books.google.com.om/ books?id=o-oDfSw562QC\&pg=PR3\&lpg=PR3\&dq=plasticine $+C$ lay+for+preparing+educational+models. [Last accessed on 2016 Feb 09].

4. Available from wikipedia:Modelling clay on use of modeling clay. http://en.wikipedia.org/wiki/Modelling_clay on use of modeling clay. [Last accessed on 2016 Feb 02].

5. Koul AR, Patil RK, Nahar S. Unfavourable results in free tissue transfer. Indian J Plast Surg 2013;46:247-55.

6. Speakers Give Sound Advice. Syracuse Post Standard; 1911. (page 18). Available from: https://en.wikipedia.org/wiki/A picture_is_worth_a_thousand_words. [Last accessed on $201 \overline{6}$
Feb 09].

7. Kirschner F, Kester L, Corbalan G. Cognitive load theory and multimedia learning, task characteristics and learning engagement: The current state of the art. Comput Human Behav 2011;27:1-4

8. Johnson DA, Christensen J. A comparison of simplified visually rich and traditional presentation styles. Teach Psychol 2011;38:293-7.

9. Penciner R. Does Powerpoint enhance learning? CJEM 2013;15:109-12.

10. Nayler J, Geddes N, Gomez-Castro C. Managing digital clinical photographs. J Audiov Media Med 2001;24:166-71.

11. Nayler JR. Clinical photography: A guide for the clinician. J Postgrad Med 2003;49:256-62.

12. Patil RK, Koul AR. Modified way of applying a tie-over dressing. Indian J Plast Surg 2016;49:124-6. 